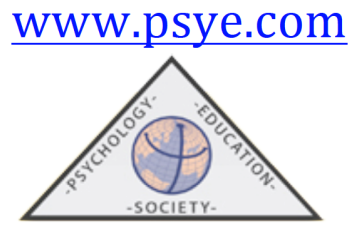

Psychology, Society, \& Education, 2020. Vol. 12(1), pp. 71-83

ISSN 2171-2085 (print) / ISSN 1989-709X (online)

Doi 10.25115/psye.v10i1.2343

\title{
Deporte e inclusión social en personas con trastorno mental grave (TMG)
}

\author{
Diego GALÁN CASADO, Joaquina CASTILLO ALGARRA \\ y Bruno GARCÍA TARDÓN
}

Universidad Camilo José Cela; Facultad de Educación de la UCJC

(Recibido el 10 de Marzo de 2019, Aceptado el 19 de Octubre de 2019)

\begin{abstract}
RESUMEN: Las personas con Trastorno Mental Grave (TMG) pueden presentar estilos de vida poco saludables (Huizing et al., 2011), lo que redunda negativamente en su salud física y en la propia enfermedad. Conociendo que el ejercicio físico regular mejora tanto la salud física como el estado emocional y el bienestar personal (Mullor et al., 2017), nos planteamos investigar las consecuencias de un programa deportivo en personas con TMG. Para ello seleccionamos una liga denominada Ligasame, dirigida a personas con TMG integradas en diferentes recursos de rehabilitación psicosocial de la Red de Atención a Personas con Enfermedad Mental de la Comunidad de Madrid y Asociaciones de Familiares, por ser una experiencia consolidada el tiempo que integra a 15 equipos de fútbol. El objetivo de este trabajo es conocer las consecuencias de este programa de fútbol en las personas con TMG que participan en el mismo, a través de la percepción de 26 profesionales de Ligasame que trabajan para las asociaciones o recursos integrados en la misma. En esta investigación, con un diseño cualitativo emergente (Hernández, Fernández y Baptista, 2006), se analizaron 26 cuestionarios de respuestas abiertas, diseñado ad-hoc para el estudio, destinado a estos profesionales. Los resultados obtenidos muestran que un programa deportivo para personas con TMG mejora su condición física, favorece sus relaciones sociales, mejora su autoestima, reduce sus niveles de estrés y ansiedad y potencia aspectos transversales como la utilización de las nuevas tecnologías o la igualdad de género. Asimismo, ayuda a reducir el estigma que sufren estas personas, tanto socialmente como a nivel personal y familiar.
\end{abstract}

Palabras clave: intervención socioeducativa, trastorno mental grave, programa deportivo, inclusión social, recuperación

\section{Sport and social inclusion in people with severe mental disorders (SMD)}

ABSTRACT: People with Severe Mental Disorder (SMD) may have unhealthy lifestyles (Huizing et al., 2011), which negatively impacts their physical health and the disease itself. Knowing that regular physical exercise improves both physical health and emotional state and personal well-being (Mullor et al., 2017), we plan to investigate the consequences of a sports program in people with SMD. To this aim, we selected a league called Ligasame, focused on people with SMD integrated in different psychosocial rehabilitation resources of the Network of Attention to People with Mental Illness of the Community of Madrid and Family Associations, as it is a consolidated experience that integrates 15 
football teams. The aim of this work is to find out what consequences this football programme has on the people with SMD who participate in it, through the perception of 26 professionals from Ligasame who work for the associations or resources integrated in it. In this research, with an emerging qualitative design (Hernández, Fernández y Baptista, 2006), 26 open-ended questionnaires were analyzed, designed ad-hoc for the study, aimed at these professionals. The results obtained show that a sports programme for people with SMD improves their physical condition, favours their social relationships, improves their self-esteem, reduces their levels of stress and anxiety and strengthens transversal aspects such as the use of new technologies or gender equality. It also helps to reduce the stigma suffered by these people, both socially and at a personal and family level.

Key words: socioeducational intervention, serious mental disorder, sport program, social inclusion, recovery

Correspondencia: Diego Galán Casado. Universidad Camilo José Cela; Facultad de Educación de la UCJC. E-mail: dagalan@ucjc.edu

\section{Introducción}

\section{La rehabilitación psicosocial de personas con enfermedad mental}

Hablar de rehabilitación psicosocial implica un conjunto de actuaciones destinadas a asegurar que una persona con una discapacidad producida por una enfermedad mental grave pueda llevar a cabo las habilidades físicas, emocionales, intelectuales y sociales necesarias para vivir en la comunidad con el menor apoyo posible por parte de los profesionales (IMSERSO, 2007). La problemática de las personas con trastorno mental grave (TMG) "no se reduce a la sintomatología psicopatológica que muestran, sino que afecta asimismo a otros aspectos, como su funcionamiento psicosocial y su participación en la comunidad" (Sobrino y Rodríguez, 2008, p. 16).

En España, la Ley General de Sanidad de 1986 propició la aparición de los primeros dispositivos de rehabilitación a partir del programa de Servicios Sociales Alternativos a la Institucionalización Psiquiátrica (1988-1995). Asimismo, la intervención a través del deporte constituye un eje fundamental para conseguir la recuperación e inclusión social y más si se tiene en cuenta que el sedentarismo se sitúa como una las principales dificultades que presentan las personas con TMG.

\section{Deporte y personas con TMG}

"La salud física de los individuos con esquizofrenia es considerablemente peor si lo comparamos con la población que no padece ningún trastorno de estas características" (Soundy, Roskell, Stubbs, Probst y Vancampfort, 2015, p. 2). A su vez, existen determinadas enfermedades mentales y situaciones en las que la inactividad física es extrema. Por ejemplo, el paso por el hospital tras una crisis psicótica genera abulia y fobia social (Villegas, López, Martínez, Luque y Villegas, 2004), lo que dificulta la capacidad de la persona para participar activamente en otros contextos sociales. Esto que, unido a "estilos de vida no saludables, influenciados por factores relacionados con la propia enfermedad" (Huizing et al., 2011, p. 11), pueden acarrear la aparición de otras patologías de carácter somático. 
Varias investigaciones han señalado que "la práctica deportiva podría mejorar determinados síntomas, como la apatía, la falta de iniciativa, la baja autoestima, las alteraciones del sueño y el aislamiento social" en personas con TMG (López-Pardo, 2014, p.1). Para que esto se produzca, la práctica deportiva deberá tener presentes los "intereses de grupos particulares, incluidas las preferencias por los tipos de actividad, así como el apoyo y las barreras percibidas" (Ussher, Stanbury, Cheeseman y Faulkner, 2007, p. 405) para la construcción de identidades tanto personales como colectivas.

El deporte orientado a las personas con TMG, está generando "un cambio en la forma de atender sus necesidades, abriendo el abanico de posibilidades a una atención multidisciplinar" (García-Pérez, Peña-Calvo y Torío-López, 2016, p. 172). Esta realidad implica entender la actividad física como un instrumento de normalización social con el que poder potenciar la estimulación, recuperación o refuerzo de aspectos de carácter transversal, combatiendo "la falta de información y la ignorancia que llevan al pesimismo sobre la capacidad y posibilidad de inclusión social" (Ceballos y Casanova, 2017, p. 126).

Teniendo estos objetivos presentes, surgieron distintas iniciativas que consideran el deporte como parte del tratamiento de las personas con TMG para conseguir su integración social. Entre estas destaca el proyecto Insport, finalizado en 2017 y que estaba inscrito en el marco del Programa Erasmus + de la Unión Europea; en él participaron nueve países europeos: Bélgica, Bulgaria, Francia, Alemania, Hungría, Italia, Portugal, España y el Reino Unido (Insportproject, 2018).

Dentro del territorio español encontramos programas similares, llevados a cabo por diferentes entidades que trabajan la recuperación de las personas con TMG a través del deporte y que tienen como objetivo principal utilizar la actividad física como instrumento para su integración social, recuperación y lucha contra la discriminación. Todas forman parte de la Asociación Española Deportiva para la Integración y la Recuperación (AEDIR), cuya finalidad es contribuir al desarrollo integral y mejorar la calidad de vida de las personas con discapacidad y/o dependencia consecutiva a un trastorno mental grave (AEDIR, 2014). Una de estas entidades es Sportsame, una agrupación de profesionales en la Comunidad de Madrid, que cuenta entre sus actividades con una liga de fútbol sala prosalud mental denominada Ligasame, en la que participan recursos de rehabilitación psicosocial de la red de Atención a Personas con Enfermedad Mental dependientes de la Consejería de Asuntos Sociales y Familia y Asociaciones de Familiares, destinados al trabajo con este colectivo (Galán-Casado, CastilloAlgarra y García-Tardón, 2018).

\section{METODOLOGÍA}

Teniendo como objetivo principal conocer las consecuencias de un programa deportivo en las personas con TMG que participan en el mismo, ha sido seleccionado como objeto de estudio el programa de fútbol Ligasame, cuyo análisis se ha desarrollado a través de la opinión de los diferentes profesionales que trabajan en dicho programa. 


\section{El programa de fútbol}

Ligasame cuenta actualmente con 15 equipos que disputan su campeonato liguero los jueves y viernes, de octubre a mayo, en las Instalaciones de Canal de Isabel II de la Comunidad de Madrid. Esta liga desarrolla una metodología activa y participativa, cuya pretensión es empoderar a los participantes, haciéndolos agentes activos del proyecto, teniendo en cuenta sus inquietudes y sugerencias de cambio, mediante su integración en el comité de disciplina, en las reuniones de delegados, en el cuerpo de árbitros encargados de velar por el cumplimiento de las normas del juego, etc. Asimismo, Ligasame cuenta con una línea de trabajo activo contra el estigma a partir de la organización de diferentes jornadas abiertas a la comunidad.

Los profesionales que trabajan con los deportistas en Ligasame son de distintas disciplinas: educadores sociales, psicólogos, terapeutas ocupacionales, trabajadores sociales y voluntarios; estos últimos reciben una formación previa desde el centro específico o asociación en la que se insertan. Todos aportan estructura a las actividades deportivas, adaptándolas a las necesidades interpersonales y grupales de las personas con TMG que atienden.

\section{Participantes}

El procedimiento de selección de la muestra de profesionales ha sido no probabilístico, participando un total de 26 personas, buscando una representatividad de los diferentes profesionales implicados, para conseguir una información lo más completa y exhaustiva posible. Finalmente accedieron a participar de manera voluntaria dos trabajadores sociales, dos terapeutas, dieciséis educadores (presentan diferentes roles dentro de su trabajo como educadores y no han sido identificados para garantizar su anonimato. Su mayor prevalencia respecto al resto de profesionales es debido a que son los encargados principales del desarrollo de las intervenciones socioeducativas) tres psicólogos y tres voluntarios (Tabla 1). Los profesionales se han enumerado del 1 al 26 para identificarlos.

Tabla 1. Profesionales participantes en la investigación

\begin{tabular}{lc} 
Profesión-cargo de los informantes & $\mathbf{N}^{\mathbf{0}}$ \\
\hline Trabajadores sociales & 2 \\
Voluntarios & 3 \\
Terapeutas & 2 \\
Educadores & 16 \\
Psicólogos & 3 \\
TOTAL & $\mathbf{2 6}$ \\
\hline
\end{tabular}

\section{Instrumentos y procedimiento para la recogida y análisis de los datos}

La investigación ha sido llevada a cabo con un diseño cualitativo emergente (Hernández, Fernández y Baptista, 2006) en el que se analizaron los datos provenientes de un cuestionario de respuestas abiertas diseñado ad-hoc para el estudio, destinado a estos profesionales. Las dos 
primeras preguntas hacían referencia a la función y rol del profesional en el recurso y en el equipo; el resto indagaban acerca de la consideración que tenía el programa de fútbol para profesionales, familiares, los recursos o asociaciones a las que pertenecían y para los propios usuarios o personas con TMG, y sobre las consecuencias que conllevaba la participación en el mismo para estas personas.

Los participantes fueron informados de que su participación sería anónima y que los datos obtenidos serían utilizados exclusivamente para la misma, así pudieron prestar su consentimiento informado (Gibbs, 2012). La cumplimentación del cuestionario se realizó a través de un formulario on-line, utilizando la plataforma de Google Docs. El análisis de los datos tuvo un componente eminentemente cualitativo.

\section{Resultados}

\section{Ligasame, mucho más que jugar al fútbol}

Los profesionales no perciben esta liga como un simple juego de fútbol, adjudicándole objetivos mucho más amplios orientados a la recuperación, lo que coincide con los resultados que obtuvo Martínez de Haro aplicando un programa de educación física con pacientes psiquiátricos agudos en 1986.

Veintiuno de los veintiséis profesionales entrevistados manifiestan que esta liga es muy importante para estos centros como herramienta de tratamiento de las personas que atienden en los mismos; incluso este programa deportivo es percibido como una parte primordial y necesaria en la consecución de los objetivos que persigue cualquier intervención socioeducativa destinada a la rehabilitación psicosocial. (Añaños-Bedriñana, 2012; Fernández-Catalina y Ballesteros-Pérez, 2017).

... "Es fundamental en nuestro centro, ya que es una gran herramienta que ayuda en la rehabilitación de nuestros usuarios" (psicólogo 3).

... "Es una actividad que se considera muy importante por el gran abanico de aspectos que permite trabajar desde la práctica deportiva a las relaciones sociales, trabajo en equipo, tolerancia a la frustración, planificación de tareas, utilización de recursos comunitarios, recuperación de actividades significativas...” (educador 11).

En este sentido, tres de los profesionales afirman que sus recursos consideran la liga de fútbol como una actividad "importante", aunque les parece insuficiente.

... "Es una actividad importante, aunque a veces, desde la dirección del centro no se ha priorizado esta actividad como se merecería" (educador 3).

... "... no se apoya mucho desde el recurso, aunque se respeta porque estoy directamente implicado en el equipo" (educador 15).

Frente a estas respuestas, otros dos profesionales declaran abiertamente que sus centros no dan ninguna importancia a esta liga. Estas dos respuestas "negativas" proceden de los dos únicos terapeutas ocupacionales entrevistados, quienes alegan incompatibilidad entre los objetivos de Ligasame y el trabajo en el recurso.

... "No (no es importante). Consideran que sólo se trabajan objetivos psicosociales y que son incompatibles con los del recurso" (terapeuta ocupacional 1). 
Sin embargo, ambos muestran su desacuerdo con esta situación y enfatizan en la importancia del deporte como medio para trabajar múltiples competencias encaminadas a la consecución de un estilo de vida normalizado (Castillo-Algarra, Galán-Casado y GarcíaTardón, 2017).

... "En mi opinión es un error... el colectivo con el que trabajamos y las pautas de derivación implican que debemos trabajar competencias transversales a todos los oficios para mejorar la empleabilidad. La actividad de Ligasame incide con gran impacto sobre esas competencias" (terapeuta ocupacional 2).

\section{Luchar contra la enfermedad, marcando goles}

Pero, ¿qué es lo que hace que la participación en Ligasame sea tan valorado por los recursos y sus profesionales? En primer lugar, el pertenecer y jugar en este programa de fútbol aporta a los usuarios beneficios físicos: aumento de la movilidad y mejora de la forma física en general, acompañado de la adquisición de hábitos saludables.

... "He percibido avances en todas estas áreas; hábitos de salud, autocuidado..." (trabajador social 1).

... "He percibido avances en lo relativo a la adquisición de competencias como higiene... mejora de dominios de salud..." (terapeuta ocupacional 2).

... Estos beneficios físicos son reconocidos por los propios usuarios “...ellos mismo te dicen que se sienten mejor a nivel físico y que liberan pensamientos negativos" (educador 3).

Junto con estos beneficios aparecen otros a nivel psicológico, que ayudan a combatir la apatía y el aislamiento (Vancampfort et al., 2012), síntomas frecuentes de las personas con TMG.

... "Les motiva, ayuda a romper su sedentarismo, su apatía... a tener en cuenta al otro ..." (educadora 16).

Otros importantes beneficios psicológicos son: aumento de la autoestima, autocontrol, autoconfianza y empoderamiento personal (Soundy, Kingstone y Coffee, 2012).

... “Están más motivados, son más autónomos, capaces de tomar decisiones que antes no tomaban, han mejorado las relaciones sociales, son más abiertos con el resto del mundo y por supuesto, han mejorado sus hábitos alimenticios y en general de salud, incluso llegan a afirmar que contribuye a la superación de miedos" (educador 1).

... "Esta actividad les ha ayudado a crecer como personas y ganar confianza" (educador 12).

Además, les ayuda a combatir y gestionar el estrés (Paluska y Schwenk, 2000), los pensamientos negativos; proporciona alegrías y aumenta su tolerancia a la frustración, haciéndoles más fuertes psicológicamente

... "Personas que empezaron a participar tras un gran esfuerzo motivador y esfuerzo personal debido al aumento de la sintomatología ante situaciones que les generaban estrés ahora son participantes activos del equipo y verbalizan disfrutar de la actividad y haber aprendido a gestionar estos síntomas" (trabajador social 2).

... "Con esta actividad trabajan aspectos como la motivación, autoestima, autoconfianza, su capacidad de decisión, de resolución de problemas..." (educador 11). 


\section{Seguimos jugando, aceptando reglas y normas e interiorizando valores, con la ayuda de nuestro "entrenador"}

Un rasgo que caracteriza la vida de las personas con TMG es la dificultad para mantener una estructura en su vida diaria. Nuestros resultados evidencian que Ligasame ayuda a cumplir reglas y a mantener una vida normalizada, aspectos que coinciden con las reflexiones de Arévalo y García (2017) a la hora de intervenir con este colectivo desde una perspectiva socioeducativa.

... "Es una herramienta muy útil para trabajar el respeto a las normas" (educador 9).

... Nos dicen que les ha ayudado en sus relaciones sociales, mejora del sueño... les ayuda a llevar una vida estructurada" (educador 13).

Otros importantes valores que trabaja Ligasame: la responsabilidad y la puntualidad.

... "Es muy importante ya que se fomenta la responsabilidad, la puntualidad, el trabajo en equipo...incluso se crean lazos de amistad y relaciones entre iguales muy importantes para su desarrollo" (educador 13).

... "Se trabaja la puntualidad, convivencia, relaciones sociales" (psicólogo 2).

Asimismo, la participación de hombres y mujeres en la liga fomenta la igualdad de género y la eliminación de actitudes despectivas hacia la mujer, trabajando la coeducación de forma transversal y favoreciendo el acceso a la práctica deportiva en igualdad de condiciones (Gallego Noche, 2015).

... "Noto una disminución de pensamientos y verbalizaciones machistas al contar con figuras femeninas dentro del equipo" (voluntario 3).

La figura del "entrenador" (profesional o voluntario) que dirige la actividad se revela como fundamental; debe ser consciente de las características y problemas de las personas con las que trabaja y del objetivo principal de la actividad.

... "Los usuarios son personas individuales y multifactoriales... hay que individualizar la evaluación de la actividad y poner en valor pros y contras" (terapeuta ocupacional 2).

... "Es una actividad que depende del recurso y con ello hay un contacto diario y se ajustan todas las expectativas dando la prioridad a los objetivos más importantes" (educador 16).

Incluso debe ser capaz de contrarrestar los problemas que pueda plantear la actividad y de convertirlos en una oportunidad para desarrollar otras aptitudes de la persona, donde las relaciones basadas en la confianza resultan fundamentales (Alba y Morán, 2017).

... "Quizá el exceso de competitividad, pero para eso estamos los profesionales para rebajarlo y dejarlo en sus justos términos" (psicólogo 19).

... "Cualquier efecto negativo que pueda conllevar (la actividad) es una buena opción para trabajar otras capacidades, como la tolerancia a la frustración o el manejo de la ira; así que no creo que conlleve a situaciones negativas siempre que haya un buen profesional cerca" (voluntario 2). 


\section{El fútbol, ¿una ayuda para la inclusión social de este colectivo?}

La participación en Ligasame permite que las personas con TMG puedan formar parte de un grupo, llegando a sentirse integrados en él; esto favorece las relaciones interpersonales y el desarrollo de otras habilidades sociales, como la empatía y la asertividad. Estos beneficios son de especial relevancia, ya que la enfermedad suele dificultar la interacción social y la pertenencia a un grupo, derivando en situaciones de exclusión y aislamiento (Magallares, 2011) que obstaculizan su proceso de recuperación.

... "Es una herramienta muy útil para trabajar habilidades sociales muy difícilmente trabajables en un aula en tiempo real, como pueden ser, la empatía, la pertenencia a un grupo" (educador 9). En la misma línea se pronuncia el educador 4.

... “... se fomenta el trabajo en equipo... incluso se crean lazos de amistad y relaciones entre iguales, muy importantes para su desarrollo" (educador 13).

... “... es un entrenamiento estupendo para aprender a manejarse en grupo y favorece que las relaciones se estrechen entre los compañeros del equipo" (educador 1).

El participar en Ligasame conlleva otro beneficio sorprendente: promueve el manejo de las nuevas tecnologías y la utilización de redes sociales para comunicarse con el grupo, aprovechando las ventajas que ofrecen estos nuevos recursos (Molina et al., 2015) en una sociedad caracterizada por la información y las nuevas formas de interacción.

... "...se trabajan transversalmente muchas otras cosas muy positivas para la rehabilitación psicosocial, como el compañerismo... incluso el manejo de nuevas tecnologías ya que crean grupos whatsapp entre ellos..." (trabajador social 2).

A través de la participación en Ligasame se trabajan otras capacidades: aumenta su autonomía personal, haciendo que se desplacen por el entorno a partir del "manejo de medios de transporte" (trabajador social 1) y "la orientación por la ciudad" (educador 9). Esto contribuye a su participación activa como ciudadanos de pleno derecho.

... “... enseña el manejo de transportes, utilización de recursos comunitarios, toma de decisiones, el empoderamiento de cada uno de ellos en general, verse capaz de decidir y hacer por sí solos y con autonomía muchas de las acciones diarias que no creían poder hacer antes" (educador16).

Por último, otro de los beneficios fundamentales que más han valorado los profesionales participantes en el estudio, es la reducción del estigma. El deporte puede eliminar prejuicios y percepciones erróneas, que etiquetan a las personas con TMG como agresivos, impredecibles en su conducta, débiles, improductivos... (Ochoa et al., 2011; Cangas et al., 2017) y que, a su vez, deriva en una incurable segunda enfermedad (Mangliano et al., 2012).

... "La sociedad, ayudada por los medios de comunicación, tiene una idea de que son "'locos"", que son peligrosos, que no se integran en la sociedad, que no saben hacer nada... y esta herramienta ha demostrado a muchas personas que todo esto no es verdad" (educador 11).

Distintos investigadores establecen los beneficios de la actividad física en personas con TMG a nivel social (Mullor et al., 2017, 518). Nuestros resultados también apuntan en este sentido, siendo el deporte un elemento fundamental ante tres tipos de estigma concretos. 


\section{Estigma social}

Ligasame propicia la relación del usuario con otras personas que también padecen un trastorno mental y, lo más importante, con personas de distintos ámbitos sociales, no relacionados con la enfermedad, favoreciendo una relación normalizada donde el contacto directo es fundamental (López-Pardo, 2014).

... "Gracias a los entrenamientos en pistas comunitarias que nos permite mezclarnos con gente de fuera del ámbito de la salud mental o a iniciativas como los torneos en los que se invita a gente sin enfermedad; así se modifican falsas creencias y se reduce el estigma, permitiendo la sana convivencia y el intercambio de ideas y opiniones" (voluntario 1).

... "Es una vía potentísima de integración y de reducción del estigma, pues se trata de una actividad que otorga gran visibilidad y tiene una tremenda proyección de cara al exterior" (trabajador social 1).

... "Esta actividad les permite relacionarse en un ambiente sociocomunitario, realizar una actividad cara a cara con personas sin enfermedad mental (y muchas veces ganándoles), viendo que son personas cordiales como cualquier otra persona, que tienen intereses y necesidades igual que ellos... en definitiva, les ayuda a cambiar esas falsas creencias y no estigmatizar tanto a las personas con enfermedad mental, ya que luego existe difusión ampliando aún más este concepto a otras personas" (educador 12).

\section{Estigma familiar}

Distintos estudios establecen principalmente dos tipos de estigma en personas con TMG (Zaninotto et al., 2018), el social y el autoestigma. Nuestro trabajo de campo pone de manifiesto la existencia de un tercer tipo: el estigma familiar, menos presente en la literatura científica (Muñoz, Pérez, Crespo y Guillém, 2009). La familia, siendo un pilar fundamental en el proceso de recuperación de la persona con TMG, percibe directamente las consecuencias, problemas y miedos que genera la enfermedad; padece "los síntomas, los efectos positivos y negativos de los tratamientos (...) además de vivir su propio proceso de duelo por las implicaciones de la enfermedad como tal" (Acero, Cano-Prous y Canga, 2016, 204). Esto hace que, en muchas ocasiones, pierda la confianza en las capacidades de recuperación de su familiar, lo que termina repercutiendo negativamente en la persona con TMG, convirtiéndose en otro estigma más a combatir. Ligasame devuelve a las familias la esperanza en la rehabilitación de sus familiares, llegando a sentirse orgullosas por lo que están consiguiendo.

... "La propia familia, a veces, se sorprende muy positivamente de que sean capaces de jugar y competir, les ayuda a recuperar la esperanza en que su familiar volverá a ser el que era" (educador 16).

... "No sólo cambios en el usuario, también en las familias y amigos, ya que han ayudado a conocer la enfermedad de su familiar o de su amigo y les hace sentirse orgullosos de él, incluso tenerles en cuenta para muchas cosas que antes ni pensaban" (educador 11). 
... "Es una manera de sentir normalidad familiar, sentirse orgulloso de una persona" (psicólogo 2).

... “... genera orgullo y satisfacción en los familiares” (educador 14).

\section{Estigma de la persona hacia sí misma (autoestigma).}

Los prejuicios que la persona con TMG tiene interiorizados supone otro de los principales problemas añadido a su proceso inclusivo. Algunas investigaciones establecen que el autoestigma que sufre una persona con enfermedad mental puede variar en función de actividades y circunstancias externas (Ben-Zeev, Frounfelker, Morris y Corrigan, 2012). Ya hemos puesto de manifiesto cómo el participar en Ligasame ayuda a estas personas a recuperar su autoestima, a empoderarse. Pero, además, contribuye a reconstruir su identidad a través de la ruptura con su propio estigma.

... "Fundamentalmente para reducir su propio estigma, al darse cuenta que son capaces de realizar una actividad exigente, de volver a hacer lo que en su día hicieron, deporte, y deporte en equipo" (educador16).

... “... perciben que les viene muy bien para mejorar todos los objetivos antes mencionado, verse incluidos en la sociedad en general como uno más, realizando las mismas actividades que el resto de la sociedad" (educador 15).

Tabla 2. Beneficios de la participación en Ligasame en personas con TMG

\begin{tabular}{|c|c|c|}
\hline Físicos & Psicológicos & Sociales \\
\hline Mejora la forma física general & Combate la frustración & Trabaja el respeto a las normas \\
\hline Aumento de la movilidad & Disminución de la apatía & $\begin{array}{l}\text { Normaliza y propicia relaciones } \\
\text { sociales }\end{array}$ \\
\hline Mejora hábitos de higiene & Aumento de la autoestima & Trabaja la puntualidad \\
\hline Mejora hábitos alimenticios & Aumento del autocontrol & Mejora las habilidades sociales \\
\hline Mejora de los autocuidados & $\begin{array}{l}\text { Favorece el empoderamiento } \\
\text { personal }\end{array}$ & $\begin{array}{l}\text { Aumenta el sentimiento de } \\
\text { pertenencia a un grupo }\end{array}$ \\
\hline Mejora del sueño & Disminución del estrés & $\begin{array}{l}\text { Combate el triple estigma: social, } \\
\text { familiar y el autoestigma }\end{array}$ \\
\hline $\begin{array}{l}\text { Propicia una vida más } \\
\text { estructurada }\end{array}$ & $\begin{array}{l}\text { Disminución de los } \\
\text { pensamientos negativos }\end{array}$ & Potencia la empatía y la asertividad \\
\hline
\end{tabular}

\section{Conclusiones}

El deporte vuelve a evidenciar su potencial integrador en un colectivo que puede presentar riesgo de exclusión social, apareciendo como un instrumento que contribuye al proceso de recuperación de las personas con TMG. Mejora su estado físico y psicológico, reduce el estrés, la ansiedad y los pensamientos negativos, junto con importantes beneficios transversales: aumenta la autonomía personal, las habilidades sociales y trabaja la coeducación. Siendo el estigma uno de los principales problemas de este colectivo, hemos identificado un tercer estigma, además del social y del autoestigma: el familiar. El deporte aparece como una potente herramienta para erradicarlo en estos tres ámbitos. La implicación de los recursos en el 
programa deportivo y la formación e implicación de los profesionales y voluntarios involucrados en el mismo aparecen como clave para su éxito.

Consideramos que los resultados obtenidos podrán ser extrapolados a programas deportivos similares, siempre que tengan los mismos objetivos, esto es, utilizar el deporte -en nuestro caso, el fútbol- como una herramienta para la recuperación psicosocial de la persona; objetivos que deben compartir igualmente los profesionales y voluntarios involucrados en los mismos.

Además, resulta necesario aumentar este tipo de iniciativas desde una perspectiva socioeducativa, que tenga en cuenta, al igual que Ligasame, fundamentos basados en el proceso de acompañamiento y en la utilización de espacios de intervención normalizados que permita a este colectivo conseguir mejorar su proceso inclusivo. Finalmente, consideramos necesario seguir profundizando en la investigación sobre deporte y enfermedad mental, analizando los diferentes programas deportivos ya existentes destinados a personas con TMG, de dentro y fuera de España.

\section{REFERENCIAS}

Alba Gómez, S. y Morán de Castro, M. C. (2017). Las educadoras y educadores sociales en el ámbito de salud mental en Galicia. Revista de Educación Social, 25, 219-235.

Acero González, A. R., Cano-Prous, A., y Canga, A. (2016). Experiencias de la familia que convive con la enfermedad mental grave: un estudio cualitativo en navarra. Anales del Sistema Sanitario de Navarra, 39(2), 203-212.

AEDIR. (2014). AEDIR. Memoria de actividades 2014. Extraído el 31 de Enero de 2019 desde http://www.aedir.es/descargas/category/8-memorias_anuales.html

Añaños-Bedriñana, F. (2012). Pensamiento y acción socioeducativa en Europa y España. evolución de la pedagogía y educación social. Revista historia de la educación latinoamericana, 14(18), 119-138.

Arévalo Blázquez, D. y García Rico, S. (2017). Comprender y ejercer la ciudadanía como educadores. Revista de Educación Social, 24, 1211-1220.

Cangas, A., López, A., Orta, A., Gallego, J., Alias, A., Soriano, C. y Piedra, J. A. (2017). "Inclúyete": Programa socioeducativo e inclusivo para personas con Trastorno Mental Grave y estudiantes en la Universidad de Almería. Revista Edupsykhé, 16(1), 86-97.

Castillo-Algarra, J., Galán-Casado, D. y García-Tardón. (2017). Deporte y Trastorno Mental Grave. El caso de LIGASAME y otras iniciativas en España. Revista Edupsykhé, 16(1), 54-65.

Ben-Zeev, D., Frounfelker, R., Morris, S. B. y Corrigan, P. W. (2012). Predictors of SelfStigma in Schizophrenia: New Insights Using Mobile Technologies. Journal of Dual Diagnosis, 8(4), 305-314.

Ceballos, I. y Casanovas, R. (2017). La función y el valor del deporte en AVIFES. Educació Social. Revista dìntervenció Socioeducativa, 65, 124-140.

Fernández-Catalina, P. y Ballesteros-Pérez, F. (2017). La rehabilitación psicosocial de personas con trastorno mental: un modelo para la recuperación e integración comunitaria. Revista Edupsykhé, 16(1), 15-36. 
Galán Casado, D., García-Tardón, B. y Castillo-Algarra, J. (2018). Voluntariado, actividad física y enfermedad mental. Una experiencia desde Ligasame. En Llopis Goig, R. y Sola Espinosa, I. (Comps.). Integración social y deporte. Investigación y estudio de casos (pp. 47-55). Valencia: Nau LLibres.

Gallego Noche, B. (2015). La igualdad de género en la práctica deportiva y su contribución a la educación para la ciudadanía y la democracia. Journal for Educators, Teachers and Trainers, 5(3), 191-203.

García-Pérez, O., Peña-Calvo, J. V. y Torío-López, S. (2016). Atención socioeducativa y trastorno mental severo: la vivienda como base de intervención. Convergencia, 23(72), 171-197.

Gibbs, G. (2012). El análisis de datos en investigación cualitativa. Madrid: Ediciones Morata.

Hernández, R., Fernández, C. y Baptista, P. (2006). Metodología de la investigación. México: McGraw Hill.

Huizing E., Padilla Obrero L., Fernández Portes L., Jimeno Luque V., Zurita Márquez I., Muñoz Bellerín J. y de la Fuente, R. (2011). Promoción de la salud en personas con trastorno mental grave: análisis de situación y recomendaciones sobre alimentación equilibrada y actividad física. Sevilla. Consejería de Salud.

IMSERSO. (2007). Modelo de centro de rehabilitación psicosocial. Madrid: Ministerios de Educación, Política Social y Deporte.

Insportproject (2018). Insport +. Promoting social inclusion of persons with mental disabilities through sport. Extraído el 20 de Febrero de 2019 desde http://insportproject.eu/project1/

Ley 14/1986, de 25 de abril, General de Sanidad.

López-Pardo, A. (2014). Deporte en personas con trastornos mentales graves: buenas prácticas en Europa. En Cangas, A. J. Gallego, J. y Navarro, N (Coords). Libro de actas del II Congreso nacional de sensibilización al estigma social en salud mental y III Congreso Internacional de Actividad Física y Deporte Adaptado. Vol I. Estigma, Deporte y Salud Mental (pp. 1-11). Granada: Alborán Editores.

Magallares Sanjuan, A. (2011). El estigma de los trastornos mentales: discriminación y exclusión social. Quaderns de Psicología, 13(2), 7-17.

Magliano, L., Read, J., Patalano, M., Sagliocchi, A., Oliviero, N., D’Ambrosio, D., Campitiello, F., Guizzaro, L. y Cerrato, F. (2012). Contrarrestar el estigma hacia las personas con esquizofrenia en el ámbito sanitario: una experiencia piloto en una muestra de estudiantes italianos de medicina. Psychology, Society \& Education, 4, 169-181.

Martínez de Haro, V. (1986). Un programa de Educación Física para pacientes psiquiátricos agudos. Apunts Educació Física, 3, 37-43.

Molina Gómez, A. M., Roque Roque, L., Garcés Garcés, B. R., Rojas Mesa, Y., Dulzaides Iglesias, M. E. y Selín Ganén, M. (2015). El proceso de comunicación mediado por las tecnologías de la información. Ventajas y desventajas en diferentes esferas de la vida social. Medisur, 13(4), 481-493.

Mullor, D., Gallego, J., Cangas, A. J., Aguilar-Parra, J. M., Valenzuela, L., Mateu, J. M. y López-Pardo, A. (2017). Efectividad de un programa de actividad física en personas con trastorno mental grave. Revista Internacional de Medicina y Ciencias de la Actividad Física y el Deporte, 17(67), 507-521. 
Muñoz, M., Pérez Santos, E., Crespo, M. y Guillém, A. I. (2009). Estigma y enfermedad mental: análisis del rechazo social que sufren las personas con enfermedad mental. Madrid: Editorial Complutense.

Ochoa, S., Martínez, F., Ribas, M., García-Franco, M., López, E., Villellas, R., Arenas, O., Álvarez, I., Cunyat, C., Vilamal, S., Autonell, J., Lobo, E. y Haro, J. M. (2011). Estudio cualitativo sobre la autopercepción del estigma social en personas con esquizofrenia. Revista Asociación Española Neuropsiquiatría, 31, 477-489.

Paluska, S. A. y Schwenk, T. L. (2000). Physical activity and mental health: Current concepts. Sports Medicine, 29, 167-180.

Sobrino, T. y Rodríguez, A. (2008). Intervención social en personas con enfermedad mental grave y crónica. Madrid: Síntesis.

Soundy, A., Roskell, C., Stubbs, B., Probst, M. y Vancampfort, D. (2015). Investigating the benefits of sport participation for individuals with schizophrenia: A systematic review. Psychiatria Danubina, 27(1), 2-13.

Soundy, A., Kingstone, T. y Coffee P. (2012). Understanding the psychosocial process of physical activity for individuals with severe mental illness: a meta-ethnography. En L'Abate L (Ed.). Mental illness 2. (1-20). Croatia: Intech.

Ussher, M., Stanbury, L., Cheeseman, V. y Faulkner, G. (2007). Physical Activity Preferences and Perceived Barriers to Activity Among Persons With Severe Mental Illness in the United Kingdom Services. Arlington, 58, 405.

Vancampfort, D., Probst, M., Helvik Skjaerven L., Catalán-Matamoros, D., LundvikGyllensten, A., Gómez-Conesa, A., Ljtema, R. y De Hert, M. (2012). Systematic review of the benefits of physical therapy within a multidisciplinary care approach for people with schizophrenia. Physical Therapy, 92, 11-23.

Villegas, I., López, J., Martínez A. B., Luque A. y Villegas J. A. (2004). Actividad física en la enfermedad mental. Revista Digital, 76. Extraído el 25 de 2019 desde http://www.efdeportes.com/

Zaninotto, L., Qian J., Sun Y., Bassi G., Solmi M. y Salcuni S. (2018). Gender, Personality Traits and Experience With Psychiatric Patients as Predictors of Stigma in Italian Psychology Students. Frontiers in Public Health, 6(362), 1-10. 